\title{
Project Title: Chemistry of Actinides in Molten Glasses and Its Correlation to Structural Performance of Solid Glasses: Filling the Knowledge Gap (Project 81926)
}

Publication Date: June 15, 2002

Principal Investigator: $\quad$ Sheng Dai

Chemical Sciences Division

Oak Ridge National Laboratory

Oak Ridge, TN 37831

Tel: (423) 576-7307

E-mail: $\underline{19 d @ o r n l . g o v ~}$

Co-Principal Investigators: Lynn Boatner, Ray F. Schumacher, and C. E. Barnes,

Postdocs: Shannon M. Mahurin

Progress Report:

RESEARCH OBJECTIVE

This proposal focuses on the following basic objectives to generate critical information for the following research Needs: (a) to conduct spectroscopic speciation of uranium compounds in glass-forming melts as a function of the acid-base composition of the glasses and melt temperatures, and to use these data to develop a general thermodynamic model for the dissolution of actinide species in oxidic glass matrices, (b ) to build a scientific basis for a new methodology to measure the basicity of glasses via optical spectra of in-situ immobilized actinides and to use this optical basicity as a primary actinide structure indicator for solid glass matrices, (c) to define the local environment of actinides in solid glasses via fluorescence lifetime distribution methods, (d) to correlate the above spectral "fingerprints" of actinides in solid and molten glasses with glass stability and the leaching rates of individual actinide species from a glass matrix.

\section{RESEARCH PROGRESS AND IMPLICATIONS}

This report essentially summarizes the work accomplished during the first six months of a three-year project. Since we are still in the initial stages of the project, our primary focus has been on the acquisition and installation of equipment specifically designed to meet the objectives of the proposal, particularly the spectroscopic investigation of actinides in glasses of various compositions through a wide range of temperatures. To that end, we have purchased and successfully installed a modern Raman system (Renishaw) capable of measuring Raman emission from $25 \mathrm{~cm}^{-1}$ to $4000 \mathrm{~cm}^{-1}$ with approximately $1 \mathrm{~cm}^{-1}$ resolution. The system is currently configured to use two laser lines as excitation sources: the $514.5 \mathrm{~nm}$ line from an argon ion laser and the $632.8 \mathrm{~nm}$ line from a helium-neon laser. A high-temperature stage (Linkam) was also obtained that coupled directly to the Raman system. This stage, in combination with a long working distance microscope objective, allows us to collect Raman spectra of samples up to a temperature of $1600^{\circ} \mathrm{C}$, well above the glass transition temperature, $\mathrm{Tg}$, of most types of glass. In addition, we have acquired a motorized XYZ translation stage that will enable us to obtain spectra at specific, predetermined sites within the sample. 
We have successfully completed some preliminary spectroscopic experiments on the dissolution of uranyl $\left(\mathrm{UO}_{2}^{2+}\right)$ in a glass matrix through the glass transition temperature. Raman spectra of uranium mixed with borosilicate glass (made by grinding a Pyrex microscope slide into a powder) were obtained at incremental temperature values up to approximately $1100^{\circ} \mathrm{C}$. Figure 1(a) shows the Raman spectrum of uranyl in glass at $950^{\circ} \mathrm{C}$ with the peak at $\sim 767 \mathrm{~cm}^{-1}$. At this temperature, blackbody radiation begins to affect the spectrum as indicated by the steadily increasing background. At higher temperatures, the blackbody radiation completely obscured the Raman signal. Glass melting points are generally lower than $1000^{\circ} \mathrm{C}$, so blackbody radiation should not be a significant problem during experiments involving in situ measurements of Raman spectra of actinides in molten glass. The solubility of the uranium in this particular type of molten glass was quite poor, thus producing large inhomogeneities in the uranium concentration throughout the glass melt. Even after heating at $1000^{\circ} \mathrm{C}$ for 16 hours, there was only nominal improvement of the dissolution of the uranyl in the borosilicate glass. Figure 2(b) shows the Raman spectrum, with the peak at $\sim 762 \mathrm{~cm}^{-1}$, of uranyl in glass after heating overnight.

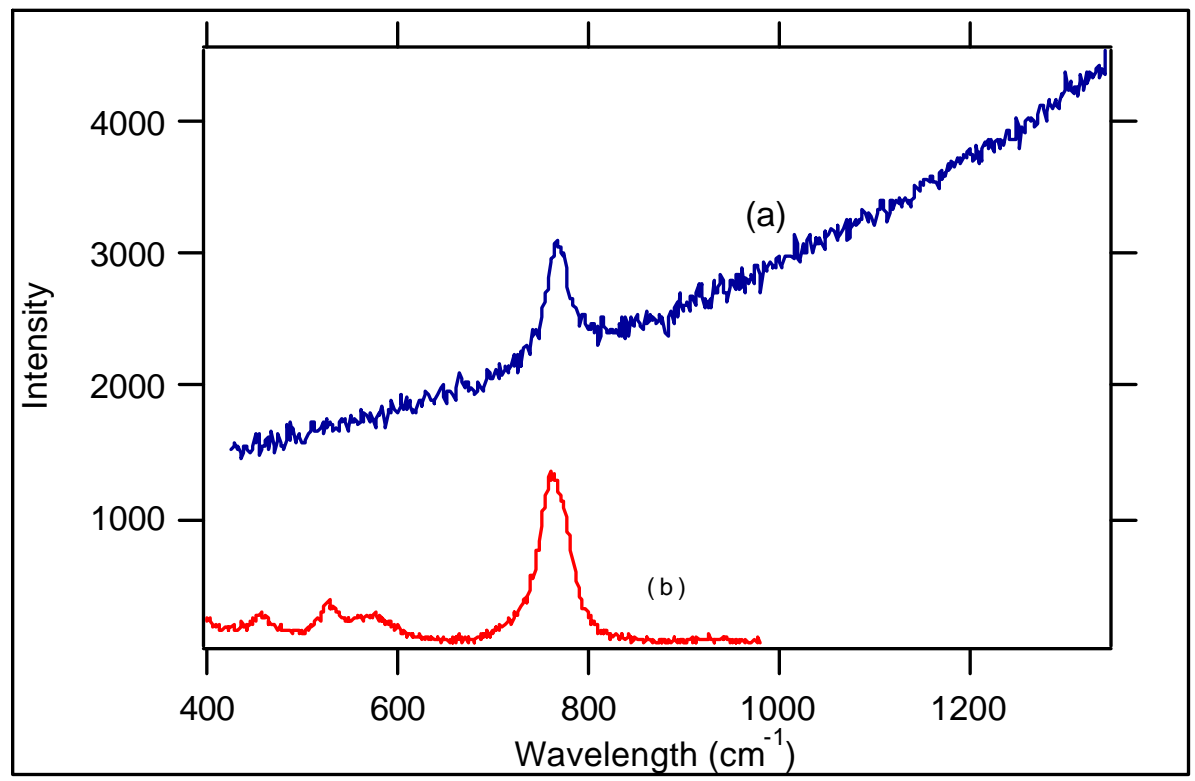

Figure 1. Raman spectra of uranyl in borosilicate glass (a) at $950^{\circ} \mathrm{C}$ and (b) at room temperature after heating at $1000^{\circ} \mathrm{C}$ for 16 hours.

We have received samples of three different frits from the Savannah River Site which have been used in the immobilization of nuclear waste material. We are currently preparing experiments to investigate the chemistry, beginning with solubility, of uranyl in molten glasses of various compositions, particularly in the frits obtained from the Savannah River Site. 